\title{
Factores de riesgo de síntomas de rinitis alérgica en adolescentes de Castellón, España
}

\author{
María Rosario Pac-Sa, ${ }^{1}$ Lidón Museros-Recatala, ${ }^{1}$ Alberto Arnedo-Pena, ${ }^{2}$ \\ Juan B. Bellido-Blasco, ${ }^{2}$ Joan Puig-Barberà, ${ }^{3}$ Adrián Artero-Sivera, ${ }^{4}$ \\ Joan-Baptista Campos-Cruañes ${ }^{5}$ e Inés Aguinaga-Ontoso ${ }^{6}$
}

Forma de citar Pac-Sa MR, Museros-Recatala L, Arnedo-Pena A, Bellido-Blasco JB, Puig-Barberà J, Artero-Sivera A, et al. Factores de riesgo de síntomas de rinitis alérgica en adolescentes de Castellón, España. Rev Panam Salud Publica. 2008;23(5):333-40.

RESUMEN Objetivo. Estimar factores de riesgo de sintomas de rinitis alérgica en adolescentes de Castellón, España.

Métodos. Estudio transversal de base poblacional a partir de la fase III del ISAAC (International Study of Asthma and Allergies in Childhood) llevado a cabo en 2002 entre adolescentes de 13 a 14 años. Se utilizó el cuestionario ISAAC para definir los casos de rinitis alérgica. Se estimaron razones de posibilidades (RP) y sus intervalos de confianza de 95\% (IC95\%) mediante modelos de regresión logística.

Resultados. La participación fue de 66,8\% (3 995 adolescentes de un total de 5 981). La prevalencia de sintomas de rinoconjuntivitis en los últimos 12 meses fue de 16,5\%, y la prevalencia de alergia nasal alguna vez, de 7,4\%. Con la regresión logística, la rinoconjuntivitis se asoció a la mujer ( $R P=1,63$; IC95\%:1,33-2,00); fumar la madre en casa ( $R P=1,32$; IC95\%:1,08-1,63); historia de sinusitis $(R P=2,02$; IC95\%:1,51-2,70), y circulación constante de camiones por la calle de residencia $(R P=1,58 ; I C 95 \%: 1,02-2,44)$. De igual manera, la alergia nasal se asoció con la historia familiar de rinitis alérgica ( $R P=2,62$; IC95\%:1,90-3,63); historia de sinusitis $(R P=2,65$; IC95\%:1,77-3,96), historia de bronquitis ( $R P=1,68$; IC95\%:1,19-2,36), y clase social, con descensos progresivos al comparar las clases superiores e inferiores.

Conclusiones. Diferentes factores de riesgo medioambientales se asociaron con el hecho de sufrir síntomas de alergia nasal; se sugiere la importancia de adecuar medidas preventivas específicas.

Palabras clave Rinitis, alergia, adolescentes, factores de riesgo, España.

Oficina Plan del Cáncer. Conselleria de Sanitat. Valencia, España.

2 Sección de Epidemiología. Centro Salud Pública. Castellón, España. La correspondencia se debe dirigir al Dr. Alberto Arnedo. L'Olivera 5 2-C Edificio Flor Azahar. Castellón de la Plana. Castellón 12005 España. T.: (34-96) 4358324. F.: (34-96) 4239491. Correo electrónico: arnedo_alb@gva.es

3 Unidad de Programas, Centro Salud Pública, Castellón, España.

4 Centro de Salud, Burriana, España.

5 SAMU de L'Alcora. Conselleria Sanitat, Castellón, España.

6 Departamento de Ciencias de la Salud, Universidad Pública de Navarra, Pamplona, España.
Hoy en día todavía no son bien conocidas las causas de la rinitis alérgica (RA), de tal manera que en el orbe se llevan a cabo investigaciones para tratar de determinarlas. Así, el protocolo ISAAC (International Study of Asthma and Allergies in Chilhood) ha permitido profundizar en la epidemiología del asma, la RA y el eczema atópico en poblaciones escolares de todo el mundo mediante un cuestionario estandarizado y una metodología común (1). En España y en algunos países de América Latina se ha podido estimar la prevalencia de síntomas de RA, distribución geográfica y tendencia (2-5), y se ha indicado la importancia del estudio de las condiciones locales para una mejor caracterización de los potenciales factores de riesgo. Sin embargo, son pocos los estudios de base poblacional de la RA que inclu- 
yan estimaciones de factores de riesgo (6-9). El objetivo del presente estudio fue estimar factores de riesgo de síntomas de RA en adolescentes de Castellón (España).

\section{MATERIALES Y MÉTODOS}

En 2002 se llevó a cabo en Castellón, España, un estudio transversal de base poblacional a partir de la fase III del ISAAC, explicado con mayor detalle por Arnedo y cols. (2). La población de estudio fueron escolares de los cursos de primer y segundo grado de educación secundaria obligatoria, esto es, adolescentes entre 13 y 14 años de edad de todos los colegios e institutos, tanto públicos como privados o concertados, de Castellón y las siguientes seis localidades próximas: El Grau, Vila-real, Almassora, Benicassim, L'Alcora y Borriol. Se solicitó permiso a las autoridades académicas y a los padres de los escolares para poder llevar a cabo la investigación. Los 46 colegios e institutos incluidos en el área de estudio participaron.

Los cuestionarios sobre síntomas de RA (anexo 1) fueron cumplimentados por los adolescentes en el colegio o instituto, y los cuestionarios de factores de riesgo de enfermedades alérgicas (anexo 2) por los padres de los adolescentes en casa. Se definió como caso de síntomas de rinoconjuntivitis (RC) en los últimos 12 meses la respuesta afirmativa a las siguientes tres primeras preguntas del cuestionario: ¿Has tenido alguna vez estornudos, te ha goteado o se te ha taponado la nariz, sin haber estado resfriado o con gripe? ¿Has tenido problemas de estornudos, te ha goteado o se te ha taponado la nariz, sin haber estado resfriado o con gripe en los últimos 12 meses? ¿Has tenido estos problemas de nariz acompañados de picor y lagrimeo en los ojos en los últimos 12 meses?

Para el caso de alergia nasal (AN), se consideró la respuesta positiva a la pregunta número seis: ¿Has tenido alguna vez alergia nasal incluyendo fiebre del heno? El cuestionario sobre factores de riesgo incluyó historia de enfermedades, historia familiar de RA, fumar en casa los padres $\mathrm{u}$ otros familiares, animales en casa, lactancia materna, asistencia a guarderías, edad de los padres, número de hermanos, orden de nacimiento, tráfico de camiones por la calle de residencia, y ocupación de los padres. Se tomó la ocupación más elevada de los padres para definir la clase social del adolescente y se obtuvieron seis grupos desde profesional (I) hasta trabajador no cualificado (VI), adoptados a partir de la clasificación inglesa (10).

\section{Análisis estadístico}

Se estimaron las prevalencias de síntomas de RC en últimos 12 meses y la prevalencia acumulada de AN. Se empleó el análisis multivariante mediante la regresión logística. Las variables dependientes fueron síntomas de RC y AN y se calcularon razones de posibilidades (RP) para estudiar las asociaciones de los potenciales factores de riesgo con intervalos de confianza de 95\% (IC95\%). Se elaboró un modelo base que incluyó las siguientes variables predictoras: edad, sexo, clase social y número de hermanos. Después, se le fueron añadiendo potenciales factores de riesgo, y se incorporaron al modelo final cuando presentaron una significación de $P<0,05$. Se estimó la bondad de ajuste mediante la prueba de Hosmers-Lomeshow (11), y todos los modelos logísticos presentaron buenos ajustes $(P>0,05)$. Para el análisis estadístico, se empleó el programa Stata Statistical 6,0, College Station, TX, Stata Corporation 1999.

\section{RESULTADOS}

Del total de 5981 adolescentes que cursaban primer y segundo grado de educación secundaria obligatoria, 5455 $(91,2 \%)$ cumplimentaron el primer cuestionario; por otro lado, se recogieron los cuestionarios completados por los padres de 3995 estudiantes, con una participación de $66,8 \%$ para ambos cuestionarios. En el cuadro 1 se describen las características de la población de estudio. Los síntomas de RC tuvie- ron una prevalencia de $16,4 \%$ y la prevalencia acumulada de $\mathrm{AN}$ fue de $7,2 \%$. La edad media de los escolares fue de 13,94 $\pm 0,83$ años con un rango de 12-17 años; 51,8\% son mujeres y $48,2 \%$ varones. Entre los diferentes factores de riesgo estudiados destacan, en relación con una mayor prevalencia de $\mathrm{RC}$, el sexo femenino, la historia familiar de rinitis alérgica y la de sinusitis y bronquitis, fumar la madre en casa, y el vivir en una calle con paso de camiones muy frecuente. En cuanto a una mayor prevalencia de AN, los factores asociados fueron clase social, historia familiar de rinitis alérgica y de sinusitis y de bronquitis, y vivir en una calle con paso muy frecuente de camiones.

En el cuadro 2 se recogen los factores de riesgo de la prevalencia de síntomas de RC por sexo y en el total de adolescentes mediante en el análisis de regresión logística. En el total se observó que la prevalencia de RC se asoció con las mujeres ( $\mathrm{RP}=1,63$; IC95\%: $1,32-2,00)$, fumar la madre en casa ( $R P=1,32$; IC95\%:1,08-1,63), historia de sinusitis ( $\mathrm{RP}=2,02$; IC95\%:1,51-2,70), y circulación constante de camiones por la calle de residencia ( $\mathrm{RP}=1,58$; IC95\%: $1,02-2,44)$. Para los varones, los factores de riesgo asociados a la prevalencia de RC fueron la historia familiar de rinitis alérgica, el fumar la madre en casa, y la historia de sinusitis. Para las mujeres, la prevalencia de RC se asoció con la mayor edad, historia de sinusitis, y circulación constante de camiones.

En el análisis de regresión logística de la prevalencia de AN en el total de adolescentes (cuadro 3), se estimaron asociaciones con la historia familiar de rinitis alérgica ( $\mathrm{RP}=2,62$; IC95\%:1,90$3,63)$, la de sinusitis $(\mathrm{RP}=2,65$; IC95\%: $1,77-3,96)$, la de bronquitis ( $\mathrm{RP}=1,68$; IC95\%:1,19-2,36), y clase social. En relación con este último aspecto, se observó un gradiente en la disminución de la prevalencia desde la clase I a la clase VI, con RP significativas al comparar la clase I con la IV $(\mathrm{RP}=0,46$; IC95\%:0,26-0,81) y la V ( $R P=0,51$; IC95\%:0,28-0,92). Para las mujeres, la prevalencia de AN se asoció con la mayor edad, clases sociales IV y V, historia familiar de rinitis alérgica y de sinusitis, haber nacido en el extranjero y 
CUADRO 1. Características de los adolescentes del ISAAC fase III. Prevalencias de síntomas de rinoconjuntivitis en los últimos 12 meses, alergia nasal y factores de riesgo, Castellón, España, 2002

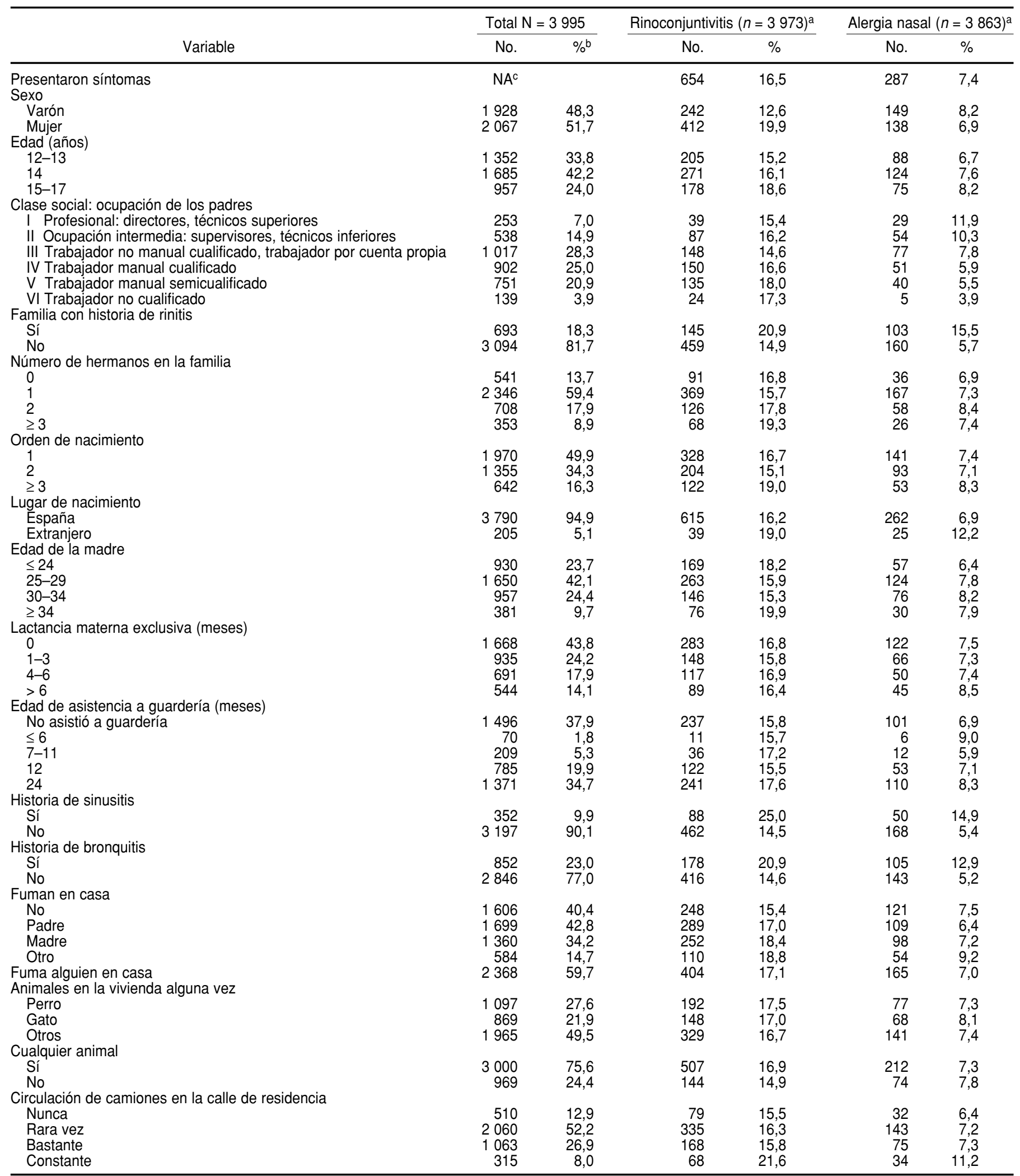

${ }^{a}$ Respuestas válidas a las cuestiones de rinoconjuntivitis $n=3973$ y alergia nasal $n=3863$.

${ }^{b}$ Porcentaje calculado sobre respuestas válidas.

${ }^{\mathrm{c}}$ No aplica. 
CUADRO 2. Factores de riesgo de síntomas de rinoconjuntivitis en los últimos 12 meses, por sexo, mediante análisis multivariante de regresión logística, Castellón, España, 2002

\begin{tabular}{|c|c|c|c|}
\hline Variable independiente & $\begin{array}{c}\text { Varón } \\
\text { RPa; IC95\% }\end{array}$ & $\begin{array}{c}\text { Mujer } \\
\text { RPa; IC95\% }{ }^{\mathrm{b}}\end{array}$ & $\begin{array}{c}\text { Total } \\
\text { RPa; IC } 95 \%{ }^{\mathrm{b}}\end{array}$ \\
\hline $\begin{array}{l}\text { Edad (años) } \\
\text { Mujer }\end{array}$ & $\begin{array}{c}1,01 ; 0,83-1,22 \\
-\end{array}$ & $\begin{array}{c}1,20 ; 1,03-1,41 \\
-\end{array}$ & $\begin{array}{l}1,11 ; 0,99-1,26 \\
1,63 ; 1,32-2,00\end{array}$ \\
\hline Historia familiar de rinitis alérgica & 1,$49 ; 1,00-2,21$ & 1,$06 ; 0,75-1,48$ & 1,$21 ; 0,93-1,56$ \\
\hline $\begin{array}{l}\text { Clase social: ocupación de los pac } \\
\text { I } \\
\text { II } \\
\text { III } \\
\text { IV } \\
\text { V } \\
\text { VI }\end{array}$ & $\begin{array}{l}1,00 \\
0,99 ; 0,48-2,04 \\
0,88 ; 0,45-1,72 \\
0,89 ; 0,45-1,76 \\
1,21 ; 0,62-2,39 \\
1,21 ; 0,45-3,21\end{array}$ & $\begin{array}{l}1,00 \\
0,96 ; 0,53-1,75 \\
0,93 ; 0,53-1,62 \\
0,98 ; 0,56-1,73 \\
1,16 ; 0,65-2,06 \\
0,82 ; 0,34-1,96\end{array}$ & $\begin{array}{l}1,00 \\
0,97 ; 0,61-1,54 \\
0,92 ; 0,60-1,41 \\
0,97 ; 0,63-1,49 \\
1,20 ; 0,77-1,85 \\
0,98 ; 0,51-1,88\end{array}$ \\
\hline $\begin{array}{l}\text { Número de hermanos en la familia } \\
0 \\
1 \\
2 \\
\geq 3\end{array}$ & $\begin{array}{l}1,00 \\
0,94 ; 0,58-1,51 \\
0,90 ; 0,50-1,60 \\
1,72 ; 0,90-3,31\end{array}$ & $\begin{array}{l}1,00 \\
0,83 ; 0.57-1,22 \\
1,06 ; 0,67-1,68 \\
1,04 ; 0,60-1,81\end{array}$ & $\begin{array}{l}1,00 \\
0,87 ; 0,64-1,16 \\
0,99 ; 0.70-1,42 \\
1,26 ; 0,83-1,92\end{array}$ \\
\hline $\begin{array}{l}\text { Historia de sinusitis } \\
\text { Fuma la madre en casa }\end{array}$ & $\begin{array}{l}2,35 ; 1,50-3,67 \\
1,44 ; 1,04-2,00\end{array}$ & $\begin{array}{l}1,85 ; 1,26-2,72 \\
1,26 ; 0,96-1,64\end{array}$ & $\begin{array}{l}2,02 ; 1,51-2,70 \\
1,32 ; 1,08-1,63\end{array}$ \\
\hline $\begin{array}{l}\text { Circulación de camiones } \\
\text { Nunca } \\
\text { Rara vez } \\
\text { Bastante } \\
\text { Constantemente }\end{array}$ & $\begin{array}{l}1,00 \\
0,84 ; 0,52-1,35 \\
0,72 ; 0,43-1,23 \\
1,06 ; 0,54-2,09\end{array}$ & $\begin{array}{l}1,00 \\
1,40 ; 0,89-2,21 \\
1,57 ; 0,97-2,55 \\
2,06 ; 1,15-3,68\end{array}$ & $\begin{array}{l}1,00 \\
1,12 ; 0,81-1,55 \\
1,15 ; 0,81-1,63 \\
1,58 ; 1,02-2,44\end{array}$ \\
\hline
\end{tabular}

a Razón de posibilidades.

b Intervalo de confianza de $95 \%$.

c I Profesional: directores, técnicos superiores; II Ocupación intermedia: supervisores, técnicos inferiores; III Trabajador no manual cualificado, trabajador por cuenta propia; IV Trabajador manual cualificado; V Trabajador manual semicualificado; VI Trabajador no cualificado.

tener tres o más hermanos. En los varones, la prevalencia de AN presentó los siguientes factores de riesgo: historia familiar de rinitis alérgica, de sinusitis y de bronquitis.

Otros factores que fueron estudiados en los modelos multivariantes para la RC y AN no presentaron asociaciones, como la edad de la madre o del padre, fumar el padre en casa, fumar otro miembro de la familia, y fumar alguien en la casa, el mes o estación de nacimiento, residir en localidades de más de 50000 habitantes, asistencia a guarderías, animales en casa alguna vez, y lactancia materna exclusiva.

\section{DISCUSIÓN}

Como hallazgos más destacados se observó que los factores de riesgo de síntomas de RC fueron el hecho de que la madre fumara en casa, el tráfico constante de camiones, ser mujer, y tener una historia de sinusitis. En cuanto a la $\mathrm{AN}$, los factores de riesgo incluyeron la historia familiar de rinitis alérgica, haber nacido en el extranjero, haber sufrido sinusitis o bronquitis, y la clase social. Se sugiere que en los síntomas de RC sería importante la contaminación del aire, tanto en el interior como en el exterior de la vivienda, y que la predisposición genética y la clase social se asociarían con la AN. La sinusitis sería un factor de riesgo de la RA.

En este estudio de base poblacional se utilizó un cuestionario de síntomas estandarizado y validado (12). Por otra parte, hubo una participación aceptable para el segundo cuestionario que, al ser llenado por los padres, permitía recopilar información más precisa acerca de los factores de riesgo, en particular de aquellos como la lactancia materna o las asistencias a guarderías. Además, es posible que se haya pro- ducido un menor sesgo de información, ya que los padres no llenaron el cuestionario de síntomas de RA por postularse que pueden tender a subestimar dichos síntomas (13).

Las limitaciones del estudio incluyen la medición de la enfermedad a través de los síntomas subjetivos, la estimación transversal de factores de riesgo, las diferencias en la participación, por sexo, edad y lugar de nacimiento, los casos detectados de curso más crónico, los potenciales sesgos de memoria de los padres y adolescentes y las diferencias socio-culturales en la comprensión y llenado de los cuestionarios (14). No se estudiaron las condiciones de la vivienda o la forma clínica, intermitente o persistente de RA que sufrían los adolescentes (15).

Se han estimado variaciones geográficas considerables en las prevalencias de RA, que podrían estar relacionadas con las condiciones locales y climáticas (16-17). Al compararla con los estudios ISAAC (18), la prevalencia de RC se situó en una zona media alta y la de AN en una baja. La mayor parte de la investigación sobre las enfermedades alérgicas se refiere al asma, cuyo estudio habitualmente es paralelo a la RA; además, existen variaciones en su definición clínica que dificultan las comparaciones entre los diferentes estudios (19). No obstante, la RA es considerada como una enfermedad multifactorial con reconocida importancia del factor hereditario, que se constata en este trabajo $(6,8,20)$. Las adolescentes sufrían mayor riesgo de síntomas de RC que los varones y las causas no son bien conocidas, aunque algunos autores han indicado una subnotificación en los hombres (21-22). El hecho de que la madre fumara en casa se asoció con sufrir síntomas de RC, y a mayor número de personas que fumaran en casa se asoció con mayor riesgo de síntomas; casi en $60 \%$ de los hogares había alguna persona que fumaba dentro de la casa. En escolares de siete años de edad, en Trinidad y Tobago (9), así como en otros estudios transversales (23-24), se ha considerado el hecho de que los padres o los abuelos fumen como factor de riesgo de rinitis, aunque hay discusión al respecto. En estu- 
CUADRO 3. Factores de riesgo de la prevalencia de alergia nasal por sexo mediante análisis multivariante de regresión logística. Castellón, España, 2002

\begin{tabular}{|c|c|c|c|}
\hline Variable independiente & $\begin{array}{c}\text { Varón } \\
\text { RPa; }^{a} \text { IC95\% }\end{array}$ & $\begin{array}{c}\text { Mujer } \\
\text { RPa; IC } 95 \%{ }^{\text {b }}\end{array}$ & $\begin{array}{c}\text { Total } \\
\text { RPa }^{\mathrm{a}} \text {; IC95\% }\end{array}$ \\
\hline $\begin{array}{l}\text { Edad (años) } \\
\text { Mujer }\end{array}$ & $\begin{array}{c}0,85 ; 0,66-1,11 \\
-\end{array}$ & $\begin{array}{c}1,41 ; 1,08-, 83 \\
-\end{array}$ & $\begin{array}{l}1,08 ; 0,90-1,29 \\
0,89 ; 0,66-1,21\end{array}$ \\
\hline Historia familiar de rinitis alérgica & 2,$35 ; 1,47-3,75$ & 3,$12 ; 1,96-4,95$ & 2,$62 ; 1,90-3,63$ \\
\hline $\begin{array}{l}\text { Clase social: ocupación de los pac } \\
\text { I } \\
\text { II } \\
\text { III } \\
\text { IV } \\
\text { V } \\
\text { VI }\end{array}$ & $\begin{array}{l}1,00 \\
1,03 ; 0,45-3,38 \\
0,71 ; 0,32-1,56 \\
0,51 ; 0,22-1,18 \\
0,60 ; 0,26-1,41 \\
0,56 ; 0,14-2,27\end{array}$ & $\begin{array}{l}1,00 \\
0,70 ; 0,31-1,57 \\
0,49 ; 0,22-1,05 \\
0,40 ; 0,18-0.90 \\
0,43 ; 0,19-1,00 \\
0,23 ; 0,48-1,69\end{array}$ & $\begin{array}{l}1,00 \\
0,83 ; 0,47-1,47 \\
0,61 ; 0,35-1,04 \\
0,46 ; 0,26-0,81 \\
0,51 ; 0,28-0,92 \\
0,43 ; 0,15-1,20\end{array}$ \\
\hline $\begin{array}{l}\text { Número de hermanos } \\
0 \\
1 \\
2 \\
\geq 3\end{array}$ & $\begin{array}{l}1,00 \\
1,48 ; 0,71-3,11 \\
1,71 ; 0,72-4,06 \\
2,43 ; 0,92-6,43\end{array}$ & $\begin{array}{l}1,00 \\
0,62 ; 0,35-1,11 \\
0,68 ; 0,33-1,41 \\
0,27 ; 0.09-0,86\end{array}$ & $\begin{array}{l}1,00 \\
0,91 ; 0,58-1,43 \\
1,01 ; 0,59-1,74 \\
0,90 ; 0,45-1,79\end{array}$ \\
\hline $\begin{array}{l}\text { Historia de sinusitis } \\
\text { Historia de bronquitis } \\
\text { Nacido en el extranjero }\end{array}$ & $\begin{array}{l}2,41 ; 1,33-4,33 \\
2,40 ; 1,52-3,81 \\
1,00 ; 0,34-2,89\end{array}$ & $\begin{array}{l}3,17 ; 1,80-5,58 \\
1,05 ; 0,62-1,78 \\
3,14 ; 1,37-7,15\end{array}$ & $\begin{array}{l}2,65 ; 1,77-3,96 \\
1,68 ; 1,19-2,36 \\
1,82 ; 0,96-3,44\end{array}$ \\
\hline
\end{tabular}

dios de prevalencia llevados a cabo en Alemania, Weiland y cols. (25) detectaron por primera vez la asociación entre tráfico de camiones y los síntomas de rinitis. En la presente investigación se encontró significación exclusivamente en la fracción de adolescentes de sexo femenino expuestas de forma muy pronunciada al tránsito de vehículos pesados. Por otra parte, la contaminación atmosférica se ha asociado con la prevalencia de RA en distintos estudios transversales (26-28). La historia de sinusitis fue otro factor de riesgo.

La prevalencia de AN presentó distintos factores de riesgo, entre ellos la historia de sinusitis cuya relación con la RA es conocida, aunque se discute su intervención como causa o consecuencia de la misma (29-30). Se ha indicado que la sinusitis y la bronquitis pueden ser factores de riesgo de AN (31-34). En estudios poblacionales (35-37) se ha encontrado que entre más elevada es la clase social, mayor es la prevalencia de AN y que esto se relacionaría con cambios en la vivienda (aislamiento, temperatura, mobiliario, limpieza, entre otros), que favorecen el incremento de ácaros en el hogar, variaciones del tamaño familiar y edad de las madres, cambios nutricionales, culturales, de recreo y de hábitos de vida, e incremento de la contaminación del aire por vehículos de motor (38). No obstante, el hecho de que las clases sociales más elevadas presenten mayor riesgo de $\mathrm{AN}$, aunque no de $\mathrm{RC}$, podría asociarse en alguna medida con un mejor acceso a los servicios médicos $\mathrm{y}$, por ende, con un diagnóstico de la enfermedad (3-4,39). Por otra parte, en un estudio de cohorte en niños suecos se observaron más riesgos de síntomas de RA en las clases inferiores (40), y podría considerarse la posibilidad de un subdiagnóstico de la AN en la población de los estratos sociales inferiores (41). El haber nacido en el extranjero se asoció a padecer $\mathrm{AN}$; sin embargo, este factor es difícil de explicar dada la diversidad de regiones de procedencia de los adolescentes (América Latina, el Magreb y Europa del Este). En las adolescentes, la prevalencia de AN fue menor cuando había más hermanos en la familia, de acuerdo con numerosos estudios (42), pero en los varones se apreció este efecto en sentido contrario, aunque sin alcanzar significación, tal vez en relación con el tamaño no muy grande de la muestra estudiada después de la estratificación. (43). No se hallaron asociaciones de las prevalencias de RC y AN con factores como edad maternal, mes o estación de nacimiento (44-45).

En cuanto a los resultados por sexo, cabe destacar que las adolescentes presentaron mayor prevalencia de RC, incrementos con la edad para $\mathrm{RC}$ y AN, incremento de RC por circulación de camiones y protección de AN a mayor número de hermanos. Esta aparente mayor afectación de las adolescentes a la RA, que surge en estas edades, ya ha sido indicada (46-47) y sus causas son desconocidas. Se especula con diferencias hormonales, olfativas, incluso de conducta y nutricionales que harían más susceptibles a las adolescentes (48-49).

En conjunto, no todos los factores de riesgo hallados pueden explicarse gracias a la llamada "hipótesis higiénica" sobre el incremento reciente de las enfermedades alérgicas (50), debatida en la actualidad (51); en este caso, parece prosperar una hipótesis más basada en cambios ambientales y culturales (52). Por otra parte, es posible que existan factores de riesgo no conocidos; como aproximación a la cuantificación del peso de los mismos, se ajustaron los modelos logísticos con los factores de riesgo estimados nulos y se calcularon las prevalencias de RC y AN. Así, para las clases sociales I y VI, en ausencia de hermanos, y una edad de 14 años, la prevalencia de RC fluctuaría entre $9,3 \%$ y $9,1 \%$ para los varones y $14,3 \%$ y $14,1 \%$ para las mujeres; la prevalencia de AN variaría entre 6,9\% y 3,1\% para varones y entre $6,2 \%$ y $2,7 \%$ para mujeres. Al comparar con las prevalencias medias de RC y AN, los factores de riesgo estimados explicarían un tercio de la RC y un $40 \%$ de la AN.

Los factores de riesgo estimados vislumbran intervenciones especificas en la prevención de esta patología, principalmente en lo que toca a medidas tendentes a la disminución de la contami- 
nación del aire tanto en la vivienda (fumar la madre en casa), como en el tráfico de vehículos de motor en las zonas habitadas, que supondrían, además de una reducción de riesgo per se, una disminución de enfermedades respiratorias como bronquitis y sinusitis (53-54). Profundizar en los factores estudiados, ampliarlos y, si fuera posible, llevar a cabo estudios de cohorte, serían las siguientes vertientes de investigación.
Agradecimientos. Los autores de este trabajo agradecen a los adolescentes y a sus padres, así como a los profesores y directores de los centros, su participación en el estudio, y a Jordi Sunyer sus comentarios al manuscrito.

\section{ANEXO 1. Cuestionario sobre síntomas de rinitis alérgica}

Todas las preguntas se refieren a problemas que te ocurren cuando NO ESTÁS resfriado o con gripe:

1. ¿Has tenido alguna vez estornudos, te ha goteado o se te ha taponado la nariz, sin haber estado resfriado 0 con gripe? SÍ/NO

2. ¿Has tenido problemas de estornudos, te ha goteado o se te ha taponado la nariz, sin haber estado resfriado o con gripe en los últimos 12 meses? Sí/NO

3. ¿Has tenido estos problemas de nariz acompañados de picor y lagrimeo en los ojos en los últimos 12 meses? Sí/NO

4. ¿En cuáles de estos meses has tenido en tu nariz estos problemas? Enero, febrero, marzo, abril, mayo, ....

5. ¿Cuántas veces los problemas de nariz te han impedido hacer tus actividades diarias en los últimos 12 meses? Nunca/pocas veces/bastantes veces/muchas veces.

6. ¿Has tenido alguna vez alergia nasal, incluyendo fiebre del heno? Sí/NO

\section{ANEXO 2. Cuestionario sobre factores de riesgo de asma, rinitis alérgica y eczema atópico}

1. ¿El niño/a toma algún medicamento (aerosol, inhalador, pastillas, jarabes, cremas, spray nasal, gotas ....) para: asma/rinitis alérgica/dermatitis atópica?

2. El médico le ha diagnosticado alguna vez al niño/a que padecía asma/neumonía/bronquitis/ sinusitis/otitis Sí/NO

3. El médico ha diagnosticado que la madre, el padre, o los hermanos del niño/a han padecido: asma/rinitis alérgica/aermatitis atópica Sí/NO

4. ¿Fuman los padres, hermanos/as u otros convivientes en casa? Padre/madre/otro (hermanos, etc.)

5. ¿Ha habido animales en casa? Alguna vez: perro, gato, otro. En la actualidad: perro, gato, otro.

6. ¿El niño/a recibió exclusivamente lactancia materna? En caso afirmativo, podría indicar ¿cuántos meses duró esta lactancia? Menos de un mes/1-3 meses/4-6 meses/más de 6 meses.

7. ¿El niño/a ha asistido a una guardería antes de los tres años? En caso afirmativo, ¿qué edad tenía cuando empezó a ir a la guardería? Seis meses o menos/ de 7-12 meses/ 1 año / 2 años.

8. ¿Tiene el niño/a hermanos/as mayores? ¿Cuántos son mayores que él/ella?

9. ¿Tiene el niño/a hermanos/as menores? ¿Cuántos son menores que él/ella?

10. ¿Cuál es la edad de los padres del niño/a? Madre/padre

11. ¿Con qué frecuencia pasan camiones por la calle donde vive el niño/a en días laborables? Nunca/rara vez/frecuente/constante

12. ¿Podría indicar la ocupación de los padres? Padre/madre

Observaciones 


\section{REFERENCIAS}

1. Ellwood P, Asher MI, Beasley R, Clayton TO, Stewart AW; ISAAC Steering Committee. The International Study of Asthma and Allergies in Childhood (ISAAC): phase three rationale and methods. Int J Tuberc Lung Dis. 2005;9(1): $10-6$.

2. Arnedo-Pena A, Garcia-Marcos L, BlancoQuiros A, Martinez A, Aguinaga I, Gonzalez $\mathrm{C}$, et al. Evolución temporal de la prevalencia de síntomas de rinitis alérgica en escolares de 13-14 años de 8 áreas españolas entre 1993-1994 y 2001-2002 según el Estudio Internacional sobre Asma y Alergias en la Infancia (ISAAC). Med Clin (Barc). 2004;123: 490-5.

3. Barraza-Villarreal A, Sanin-Aguirre LH, TéllezRojo MM, Lacasaña-Navarro M, Romieu I. Prevalencia de asma, rinitis y eczema en escolares de la ciudad de Cuernavaca, México. Salud Publica Mex. 1997;39:497-506.

4. Caussade LS, Valdivia CG, Navarro MH, Pérez BE, Aquevedo SA, Sánchez DI. Prevalencia de síntomas de rinitis alérgica y su relación con factores de riesgo en escolares de Santiago, Chile. Rev Med Chile. 2006;134(4): 456-64.

5. Borges WG, Burns DA, Felizola ML, Oliveira BA, Hamu CS, Freitas VC. Prevalence of allergic rhinitis from Distrito Federal, Brazil: comparison between ISAAC phases I and III. J Pediatr (Rio J). 2006;82:137-43.

6. Fuertes J, Meriz J, Isanta C, Pardos C, López $\mathrm{V}$, González E. Factores de riesgo de asma, alergia e hiperreactividad bronquial en niños de 6 a 8 años. An Esp Pediatr. 2001;55(3): $205-12$.

7. Soto-Quiros ME, Silverman EK, Hanson LA, Weiss ST, Celedon JC. Maternal history, sensitization to allergens and current wheezing, rhinitis and eczema among children in Costa Rica. Pedriatr Pulmonol. 2002;33:237-43.

8. Barraza A, Sanin LM, Tellez MM, Lacasaña M, Romieu I. Risk factors for asthma in schoolchildren from Ciudad Juarez, Chihuahua. J Asthma. 2003;40:413-23.

9. Monteil MA, Joseph G, Chang Kit C, Wheeler G, Antoine RM. Smoking at home is strongly associated with symptoms of asthma and rhinitis in children of primary school age in Trinidad and Tobago. Rev Panam Salud Publica. 2004;16 (3):193-8.

10. Alvarez-Dardet, Alonso J, Domingo A, Regidor E. La medición de la clase social en ciencias de la salud. Barcelona: SG Editores; 1995.

11. Hosmer DW, Lemeshow S. Applied logistic regression. New York: John Wiley \& Sons; 1989.

12. Braun-Fahrländer $C$, Wüthrich $B$, Grize $L$, Sennhauser FH, Varonier HS, Vuille JC, et al. Validation rhinitis symptom questionnaire (ISAAC core questions) in a population of Swiss schoolchildren visiting the school health services. Pediatr Allergy Immunol. 1997; 8(2):75-82.

13. Renzoni E, Forastiere F, Biggeri A, Viegi G, Bisanti L, Chellini E, et al. Differences in parental- and self-report of asthma, rhinitis and eczema among Italian adolescents. Eur Respir J. 1999;14 (3):597-604.
14. Braun-Fahrländer C, Gassner M, Grize L, Takken-Sahli K, Neu U, Stricker T, et al. No further increase in asthma, hay fever and atopic sensitization in adolescents living in Switzerland. Eur Respir J. 2004;23 (3):407-13.

15. Bousquet J, Van Cauwenberge P, Khaltaev N. Allergic rhinitis and its impact on asthma. J Allergy Clin Immunol. 2001;108 (5 Suppl): S147-334.

16. Wjst M, Dharmage $S$, André E, Norback D, Raherison C, Villani S, et al. Latitude, birth date, and allergy. PLoS Med 2005;2(10):e294.

17. Azpiri A, Gamboa PM, Fernández de Corres L, Alonso E, Escobar A, Jáuregui J, et al. Prevalence of pollinosis in the Basque Country. Allergy. 1999;54(10):1100-4.

18. Strachan DP, Sibbald B, Weiland S, AitKhaled N, Anabwani G, Anderson HR, et al. Worldwide variations in the prevalence of allergic rhinoconjuntivitis in children: the International Study of Asthma and Allergies in Childhood (ISAAC). Pediatr Allergy Immunol. 1997;8:161-76.

19. Arnedo A. Prevalencia de rinitis alérgica y eczema atópico en población escolar de Castelló [tesis doctoral]. Pamplona: Universidad Pública; 1999.

20. Wright A, Holberg C, Martinez F, Halonen M, Morgan W, Taussing LM. Epidemiology of physician-diagnosed allergic rhinitis in childhood. Pediatric. 1994;94:895-901.

21. Wieringa $\mathrm{MH}$, Weyler JJ, Van Bever HP, Nelen VJ, Vermeire PA. Gender differences in respiratory, nasal, and skin symptoms: 6-7 versus 13-14 year-old children. Acta Pediatr. 1999;80:147-9.

22. Mercer MJ, Joubert G, Ehrlich RI, Nelson H, Poyser MA, Puterman A, et al. Socioeconomic status and prevalence of allergic rhinitis and atopic eczema symptoms in young adolescents. Pediatr Allergy Immunol. 2004; 15:234-41.

23. Steen-Johnsen R, Bolle R, Holt J, Magnus P. Impact of pollution and place of residence on atopic diseases among schoolchildren in Telemark County, Norway. Pediatr Allergy Immunol. 1995;6:192-9.

24. Tsunoda K, Ohta Y, Hinogami M, Soda Y. Does passive smoking affect the incidence of nasal allergies? Am J Public Health. 1995;85: 1019-20.

25. Weiland SK, Mundt K, Rückman A, Keil U. Self-reported wheezing and allergic rhinitis in children and traffic density on street of residence. Ann Epidemiol. 1994;4:243-7.

26. Pennard-Morand C, Charpin D, Raherison C, Kopferschmitt C, Caillaud D, Lavaud F, et al. Long-term exposure to background air pollution related to respiratory and allergic health in schoolchildren. Clin Exp Allergy. 2005;35: 1279-87.

27. Bugiani M, Carosso A, Migliore E, Piccioni P, Corsico A, Olivieri M, et al. Allergic rhinitis and asthma comorbidity in a survey of young adults in Italy. Allergy. 2005;60 (2):165-70.

28. Hwang BF, Jaakkola JJK, Lee YL, Lin YC, Guo YL. Relation between air pollution and allergic rhinitis in Taiwanese schoolchildren. Respir Res. 2006; 7:23.
29. Berrettini S, Carabelli A, Sellari-Franeschini $S$, Bruschini L, Abruzzese A, Quertieri F, et al. Perennial allergic rhinitis and chronic sinusitis: correlation with rhinologic risk factors. Allergy. 1999;54:242-8.

30. Min YG, Jung HW, Kim HS, Park SK, Yoo KY. Prevalence and risk factors for perennial allergic rhinitis in Korea: results of a nation wide survey. Clin Otolaryngol. 1997;22:139-44.

31. Chen CF, Wu KG, Hsu MC, Tanq RB. Prevalence and relationship between allergic diseases and infectious diseases. J Microbiol Immunol Infect. 2001;34(1):57-62.

32. Pereira A, Maldonado JA, Sanchez JL, Gómez M, Grávalos J, Pujol E. Factores de riesgo relacionados con los síntomas respiratorios, hiperreactividad bronquial y atopia. Neumosur. 1995;7:77-82.

33. Ponsonby AL, Couper D, Dwyer T, Carmichael A. Cross sectional study of the relation between sibling number and asthma, hay fever, and eczema. Arch Dis Child. 1998;79 (4):328-33.

34. Aberg N, Sundell J, Eriksson B, Hesselmar B, Aberg B. Prevalence of allergic diseases in schoolchildren in relation to family history, upper respiratory infections and residential characteristics. Allergy. 1996;51(4):232-7.

35. Butland B, Strachan DP, Lewis S, Bynner J, Butler N, Britton J. Investigation into the increase in hay fever and eczema at age 16 observed between the 1958 and 1970 British birth cohort. BMJ. 1997;315:717-21.

36. Chen JT, Krieger N, Van Den Eeden SK, Quesenberry CP. Different slopes for different folks: socioeconomic and racial/ethnic disparities in asthma and hay fever among 173859 U.S. men and women. Environ Health Perspect. 2002;110 (Suppl 2):211-6.

37. Brabäck L, Hjern A, Rasmussen F. Social class in asthma and allergic rhinitis: a national $\mathrm{CO}^{-}$ hort study over three decades. Eur Respir J. 2005;26:1064-8.

38. Nicolaou N, Siddique N, Custovic A. Allergic disease in urban and rural populations: increasing prevalence with increasing urbanization. Allergy. 2005:1357-60.

39. Sibbald B. Epidemiology of allergic rhinitis. En: Burr ML, ed. Epidemiology of clinical allergy. Basel: Karger AG; 1993. Pp. 61-79.

40. Almqvist $C$, Pershagen G, Wickman M. Low socioeconomic status as a risk factor for asthma, rhinitis and sensitization at 4 years in a birth cohort. Clin Exp Allergy. 2005;35: 612-8.

41. Litonjua AA, Celedon JC, Hausmann J, Nokolov M, Sredl D, Ryan L, et al. Variation in total and specific IgE: effects of ethnicity and socieconomic status. J Allergy Clin Immunol. 2005;115:751-7.

42. Westergaard T, Rostgaard K, Wohlfahrt J, Kragh-Andersen P, Aaby P, Melbye M. Sibship characteristics and risk of allergic rhinitis and asthma. Amer J Epidemiol. 2005;162(2): $125-32$.

43. McKeever TM, Lewis SA, Smith C, Collins J, Heatlie H, Frischer M, et al. Sibling, multiple births, and incidence of allergic disease: a birth cohort study using the West Midlands 
general practice research database. Thorax 2001;56:758-762.

44. Kinra S, Smith GD, Jeffreys M, Gunnell D, Galobardes B, McCarron P. Association between sibship size and allergic diseases in the Glasgow Alumni Study. Thorax. 2006;61:48-53.

45. Sunyer J, Antó JM, Harris J, Torrent M, Vall O, Cullinan $\mathrm{P}$, et al. Maternal atopia and parity. Clin Exp Allergy. 2001;31:1352-5.

46. Maziak W, Behrens T, Brasky TM, Duhme H, Rzehak P, Weiland SK, et al. Are asthma and allergies in children and adolescents increasing? Results from ISAAC phase I and phase III surveys in Munster, Germany. Allergy. 2003; 58:572-9.

47. Fagan JK, Scheff PA, Hryhorczuk D, Ramakrishnan V, Ross M, Persky V. Prevalence of asthma and other allergic diseases in an adolescent population: association with gender and race. Ann Allergy Asthma Immunol. 2001;86:177-184.

48. Shusterman D, Murphy MA, Balmes J. Differences in nasal irritant sensitivity by age, gender, allergic rhinitis status. Int Arch Occup Environ Health. 2003;76:577-83.

49. Osman M. Therapeutic implications of sex differences in asthma and atopy. Arch Dis Child. 2003;88:587-90.

50. Strachan DP. Family size, infections and atopy: the first decade of the "hygiene hypothesis." Thorax. 2000;55(Suppl 1):S2-10.

51. Karmaus W, Gangur V. Does allo-immune reactivity play a role in the prenatal programming of childhood allergy? Clin Exp Allergy. 2005;35(4):405-7.

52. Maziak W. The asthma epidemic and our artificial habitats.BMC Pulmonary Medicine 2005;5:5 [publicación en línea] 2005. March 31
Hallado en: URL: http:/ / www.biomedcentral. com/1471-2466-5-5.

53. Dockery DW, Speizer FE, Stram DO, Ware JH, Spengler JD, Ferris BG Jr. Effects of inhalable particles on respiratory health of preadolescent children. Am Rev Respir Dis. 1989;139(3): $587-94$.

54. Heinrich J, Hoeslscher B, Frye C, Meyer I, Pitz $\mathrm{M}$, Cyrys J, et al. Improved air quality in reunified Germany and decrease in respiratory symptoms. Epidemiology. 2002;13:394-401.

Manuscrito recibido el 15 de febrero de 2007. Aceptado para publicación, tras revisión, el 19 de octubre 2007.

ABSTRACT Objective. To estimate the risk factor for symptoms of allergic rhinitis in adolescents in Castellón, Spain.

Risk factors for symptoms of allergic rhinitis in adolescents in Castellón, Spain

Methods. A cross-sectional population based study of Phase III of the International Study of Asthma and Allergies in Childhood (ISAAC) conducted in 2002 among adolescents from 13-14 years of age. The ISAAC questionnaire was used to define cases of allergic rhinitis. Logistic regression models were used to estimate odds ratio (OR) and their $95 \%$ confidence intervals (95\%CI).

Results. Participation was $66.8 \%$ (3 995 adolescents of the 5981 total). The prevalence of rhinoconjunctivitis symptoms in the last 12 months was $16.5 \%$ and the prevalence of nasal allergy at some point was $7.4 \%$. Logistic regression showed that rhinoconjunctivitis was associated with being female $(\mathrm{OR}=1.63 ; 95 \% \mathrm{CI}$ : $1.33-2.00)$; a mother who smokes in the home $(\mathrm{OR}=1.32 ; 95 \% \mathrm{CI}$ : $1.08-1.63)$; a history of sinusitis $(\mathrm{OR}=2.02 ; 95 \% \mathrm{CI}: 1.51-2.70)$; and living on a street with heavy truck traffic $(\mathrm{OR}=1.58$; 95\%CI: 1.02-2.44). Likewise, nasal allergy was associated with a family history of allergic rhinitis (OR $=2.62$; 95\%CI: 1.90-3.63); a history of sinusitis (OR $=2.65 ; 95 \%$ CI: 1.77-3.96); a history of bronchitis (OR $=1.68 ; 95 \% \mathrm{CI}$ : $1.19-2.36)$; and social class, with a steady decline when comparing higher classes to lower classes.

Conclusions. Various environmental risk factors were associated with the symptoms of nasal allergies, which points to the importance of implementing specific preventive measures.

Key words Rhinitis, hypersensitivity, adolescents, risk factors, Spain. 